\title{
Evaluación comprensiva del profesorado universitario
}

\author{
Sebastián Rodríguez Espinar.
}

\section{Universitat de Barcelona}

Introducción

Desde la última década de siglo estamos asistiendo a un frenesí de actividad evaluadora que, en ocasiones, desvirtúa la naturaleza y finalidad de la misma que, como dimensión científica, exige la aplicación de una determinada lógica que muy frecuentemente no es concordante con la lógica política o de control que de hecho se adopta. Algunos autores han definido esta situación como la del estado evaluativo Términos como rendición de cuentas, indicadores de rendimiento, evaluación orientada a logros, evaluación institucional, acreditación, evaluación orientada a la mejora, habilitación, homologación, etc. forman parte de un discurso que pone como gran argumento de la evaluación el logro de la calidad, de la excelencia si se da rienda suelta al verbo.

Por otra parte, cada vez hay mayor evidencia de que la evaluación del profesorado universitario exige un marco contextual de evaluación más amplio: el del centro -titulación. No de extraño, por tanto, que aparezca como un área dentro de las formulaciones de estándares, como es el caso de la Federación Mundial de Educación Médica.

Finalmente, el objetivo de esta presentación es el de intentar responder a la pregunta de si es necesaria y posible una evaluación comprensiva (global) del profesorado universitario. En la medida de nuestras posibilidades intentaremos precisar la respuesta en el contexto de la Educación Médica.

\section{A. Puntos de partida}

Cuatro puntos tomaremos en consideración: los roles y funciones del profesorado, la tensión entre docencia e investigación, el marco legal que regula la evaluación del profesorado ya el mandato de evaluación o acreditación de los propios programas de formación médica. Desde cada uno de ellos pretendemos concluir en la misma dirección: es necesario integrar armónicamente las diferentes dimensiones de actividad académica, los diferentes agentes de evaluación así como los múltiples procedimientos y estrategias que se adoptan en la evaluación del profesorado.

\section{Roles y funciones del profesorado universitario}

¿Es posible definir un único perfil de roles y funciones del profesorado universitario?. ¿Las diferentes etapas profesionales (tipo y nivel) no requieren atención a específicas tareas o actividades? Frente a una concepción única y estable del perfil de dedicación hemos de asumir la diversidad o diferente ponderación de la importancia de los diferentes roles y funciones. Parece imposible responder afirmativamente a la pregunta de si un profesor/a universitario puede en solitud (aisladamente) responder a la creciente y diferente demanda que genera el nuevo contexto universitario.

El informe de seguimiento de la Conferencia Mundial sobre Educación Superior de 1998, patrocinada por la UNESCO (Fielden (2001) señala que el profesorado universitario deberá afrontar cambios muy significativos en sus patrones de actuación. Presionado por razones financieras, deberá hacer más con menos. Atenderá a estudiantes cada vez más diversos con muy diferentes metodologías y tecnologías docentes. Por otra parte, la rendición de cuentas formará parte esencial de la propia actividad docente, debiendo dar buena cuenta el profesorado de competencias como:

- Tener un compromiso científico con la disciplina, manteniendo los estándares profesionales y estando al corriente de los avances del conocimiento. 
- Identificar y comprender las diferentes formas (vías) en que aprenden los estudiantes.

- Poseer conocimientos, habilidades y actitudes relacionadas con el diagnóstico y evaluación del alumnado a fin de ayudarles en su aprendizaje.

- Conocer las aplicaciones de las TIC al campo disciplinar, tanto desde la perspectiva de las fuentes documentales como de la metodología de enseñanza.

- Ser sensible ante las señales externas del mercado sobre las necesidades laborales y profesionales de los graduados.

- Dominar los nuevos avances en el proceso de enseñanza-aprendizaje en orden a poder manejar la doble vía, presencial y a distancia, usando materiales similares.

- Tomar en consideración los puntos de vista y aspiraciones de los usuarios de la enseñanza superior, especialmente de los estudiantes.

- Comprender el impacto que los factores de internacionalización y multiculturalidad tendrán en el currículo de formación

- Poseer la habilidad para enseñar a un amplio y diverso colectivo de estudiantes, con diferentes orígenes socioeconómicos y culturales y a lo largo de horarios amplios y discontinuos.

- Ser capaz impartir docencia, tanto a grupos numerosos como a pequeños grupos (seminarios) sin menoscabar la calidad de la enseñanza.

- Desarrollar un conjunto de estrategias para afrontar diferentes situaciones personales y profesionales.

En la actividad investigadora:

- Capacidad de generar / lograr financiación para proyectos competitivos (networking and fund raising for projects)

- Incremento de la productividad de proyectos y publicaciones. En consecuencia:

- Mejor diseño de proyectos (más dedicación con riesgo de no consecución)

- Gestión de equipos, con especial atención a estudiantes de doctorado y jóvenes investigadores.

- Gestión integral (project management), con especial énfasis en proyectos internacionales.
Esta lista de competencias, que podría ser completada con las referidas a la gestión universitaria y a la asistencia profesional (dimensión de especial consideración en el profesorado de medicina) es inviable exigirla en la individualidad de una persona, sino que será necesario la configuración de equipos de trabajo en los que pueda darse la especialización en algunas de estas competencias. Ahora bien, ante esta desafiante realidad, emerge como uno de los primeros temas de debate la relación entre la actividad docente y la investigadora.

\section{La controversia docencia-investigación}

Tema siempre en el punto de mira. En otro lugar (Rodríguez, 2003) hemos documentado ampliamente la conclusión general de que a la luz de los estudios empíricos parece existir escasa relación entre ambas actividades. Si atendemos a la perspectiva de la metodología utilizada en la evaluación de la docencia y de la investigación, también encontramos elementos que nos apuntan a la diferenciación. En el cuadro 1 presentamos los resultados de un amplio estudio realizado en USA con el apoyo de la Carnegie Foundation. Dos puntos desearíamos destacar:

- La desigual toma en consideración de los mutuos impactos entre docencia e investigación. Mientras que el impacto de la docencia en la investigación sólo es considerado por el 15\% ( baja al $10 \%$ en las research universities), el inverso es considerado por el $42 \%$ de las instituciones encuestadas. Bien es cierto que aparece una gran discrepancia entre las universidades orientadas a la investigación y las comprensivas (con mayor atención a la enseñanza).

- El uso del peer review (interno o externo a la institución), estrategia clave para el reconocimiento del valor de cada dimensión por parte de la comunidad de profesorado, presenta un mayor uso en el caso de la investigación que en la docencia.

Sin duda alguna, estos y otros hallazgos e intuiciones han llevado a numerosos analistas y críticos de la educación superior a lanzar una voz de alerta sobre la necesidad de revitalizar la función docente del profesorado universitario. Bárbara Millis (1994) defiende en un interesante artículo el por qué ya no puede espe-

Cuadro 1. Metodologías de evaluación de la docencia y de la investigación

METODOLOGIA DE EVALUACIÓN DE LA DOCENCIA

¿Cuáles de los siguientes métodos de evaluación son generalmente usados en su institución a afectos de promoción y tenure?

\begin{tabular}{|c|c|c|c|c|}
\hline & Total & Research Univ. & Ph.D. Univ. & Compreh.Univ \\
\hline Autoevaluación & $82 \%$ & $66 \%$ & $74 \%$ & $83 \%$ \\
\hline Peer Review (Aula) & $58 \%$ & $40 \%$ & $41 \%$ & $63 \%$ \\
\hline Peer Review (Plan docente) & $62 \%$ & $45 \%$ & $56 \%$ & $70 \%$ \\
\hline Encuesta estudiantes (Aula) & $98 \%$ & $100 \%$ & $96 \%$ & $98 \%$ \\
\hline Encuesta estudiantes (Tutoría) & $24 \%$ & $24 \%$ & $14 \%$ & $17 \%$ \\
\hline Rendimiento estudiantes & $24 \%$ & $17 \%$ & $18 \%$ & $24 \%$ \\
\hline Continuing Student Interest & $34 \%$ & $24 \%$ & $18 \%$ & $33 \%$ \\
\hline Opinión de los graduados & $31 \%$ & $24 \%$ & $14 \%$ & $30 \%$ \\
\hline Impacto Docenc. en la Investigación & $15 \%$ & $10 \%$ & $14 \%$ & $14 \%$ \\
\hline Impacto Docenc. en Transf. de Tecnol. & $14 \%$ & $12 \%$ & $16 \%$ & $16 \%$ \\
\hline
\end{tabular}

METODOLOGIA DE EVALUACIÓN DE LA INVESTIGACIÓN

¿Cuáles de los siguientes métodos de evaluación del profesorado son generalmente usados en su institución?

\begin{tabular}{|c|c|c|c|c|}
\hline & Total & Research U. & Ph.D. Univ. & Compreh.Univ \\
\hline $\mathbf{N}^{\circ}$ publicac. pond. por tipo & $54 \%$ & $87 \%$ & $90 \%$ & $58 \%$ \\
\hline Peer Review (especif. crit. cualit.) & $44 \%$ & $86 \%$ & $66 \%$ & $41 \%$ \\
\hline Peer Review (Otras universidades) & $39 \%$ & $100 \%$ & $71 \%$ & $25 \%$ \\
\hline Peer Review (Propia universidad) & $73 \%$ & $93 \%$ & $83 \%$ & $70 \%$ \\
\hline Autoevaluación (Informe) & $77 \%$ & $69 \%$ & $72 \%$ & $75 \%$ \\
\hline Particip. estudiantes en proyectos & $37 \%$ & $25 \%$ & $32 \%$ & $36 \%$ \\
\hline Impacto Invest. en la Docencia & $42 \%$ & $21 \%$ & $24 \%$ & $46 \%$ \\
\hline Impacto Invest. en Transf. deTecnol. & $34 \%$ & $36 \%$ & $36 \%$ & $37 \%$ \\
\hline
\end{tabular}


rar más el atender y realzar el valor de la docencia (ha de evitarse que el profesorado continúe "flight from teaching"). Tras enumerar múltiples argumentos de autoridad (Astin, Boyer, Cross, Hutchings, Kuhn , Seldin, etc) y analizar los cambios en la tipología de estudiantes que acceden a la enseñanza superior, el impacto de las TIC y el cambio de paradigma en el proceso de enseñanza-aprendizaje, la autora concluye con la necesidad de que se instauren programas de desarrollo profesional que respondan creativa y responsablemente a las nuevas necesidades..

Hace ya una década, Schuster (1993) planteaba una cuestión que pueda llamar la atención: ¿Por qué un profesor ha de repartir a partes iguales su actividad docente e investigadora? ¿Por qué no se toma en consideración lo que le gusta hacer, en lo que rinde más? ¿Acaso no podría plantearse su contrato por periodos? Tal vez enarbolar este planteamiento en nuestro contexto sería peligroso. ¿Cuántos optarían por la exclusividad en la docencia y abandonarían la presión de la investigación? Tal vez serían más los que volarían de la docencia al paraíso del descubrimiento de la verdad. Podría darse la sorpresa si previamente se estableciera el verdadero concepto de investigación, o al menos se introdujera en nuestro vocabulario la interesante distinción inglesa entre scholarship y research.

Sea como fuere, sí que desde la perspectiva evaluadora, como señala Arreola (2000), es totalmente necesario explicitar los términos de dedicación: no es posible abordar una evaluación global del profesorado sin la existencia de un contrato de funciones $y$ tareas en el que debe especificar la ponderación de cada una de ellas y para cada una de las situaciones, tipologías o individualidades de profesorado. Contra este contrato (conforme al mismo) ha de plantearse una evaluación comprensiva orientada al reconocimiento, promoción e incentivación.

\section{Los marcos legales}

El nuevo marco legal de la Ley Orgánica de Universidades L.O.U.- (Ley 6/2001, de 21 de Diciembre) ha convulsionado el status quo de las dos últimas décadas, planteando una situación bastante inusual en los sistemas universitarios de nuestro entorno. Examinemos el antes y el ahora (aún no operativo) y podrá comprobarse la dificultad de lograr una evaluación comprensiva si ni se genera una cultura de cooperación y complementariedad entre todos los que tienen algo que decir, al menos desde el punto de vista legal. No hemos de olvidar que la institución (universidad) aparece con frecuencia en segundo término, situación inexplicable desde los planteamientos teóricos de una evaluación integral del profesorado universitario.

\subsection{La experiencia inmediata}

(Art. 35 y 39 de la LRU)

- Acceso a los cuerpos de profesorado universitario

- Evaluación integrada y unidad de valoración: un único acto y Comisión.

- La trayectoria académica, docente e investigadora y la defensa de un Proyecto Docente como criterio primario.

- Énfasis en el dominio de la materia en el caso de TEU,TU y CEU

. Exposición y debate de un tema del programa presentado por el candidato y elegido por éste.

- Énfasis en la investigación en el caso de C.U.

Defensa de un Proyecto Docente e Investigador

Presentación y discusión de un trabajo original de investigación

- Intencionalidad de tomar en consideración la valoración interna de la universidad

"Los Estatutos de la Universidad dispondrán los procedimientos para la evaluación periódica del rendimiento docente y científico del profesorado, que será tenido en cuenta en los concursos a que aluden los artículos treinta y cinco y treinta y nueve, a efectos de su continuidad y promoción" (L.R.U. art. 45.3)

- Varios hechos claramente contrastados:

No ha cristalizado un concepto y estructura homogénea del Proyecto Docente.
Escasa transparencia metodológica en la definición de criterios e indicadores y sus ponderaciones y estándares de calidad por parte de las Comisiones de acceso.

Nula efectividad, dada su inadecuación o ausencia, de los informes de evaluación interna de los responsables académicos.

"si estos no llegan a tiempo no se tendrán en consideración" (art. 8.4 del R.D. 1427/1986).

- Retribuciones al profesorado (incentivos por docencia e investigación)

- R. D. 1086/1989 de 28-8-89 sobre quinquenios y sexenios

- Resolución de 20-6-90 del Consejo de Universidades (BOE de 306-90) que establece los criterios de evaluación.

- Diferente jurisdicción en la valoración de la docencia (Universidad) e investigación (Comisión nacional). Decisión acordada por la comunidad universitaria, pero no establecida por derecho en la LRU.

- Varios hechos claramente contrastados:

Generación de dos culturas muy diferentes de evaluación, produciéndose una visión dividida de la calidad del profesorado universitario.

La concesión de complementos docentes a quienes no ejercían de docentes ha desvirtuado (eliminando) el proceso de evaluación docente.

Las encuestas de opinión del alumnado sobre la actuación docente del profesor se han convertido en el único referente evaluativo de la calidad del mismo.

Ausencia clara de una evaluación orientada a la mejora de la actividad docente.

\subsection{El nuevo marco (LOU)}

\section{- Integrar docencia e investigación.}

- Los artículos 33.3 y 40.3 señalan que tanto la actividad y dedicación docente (y la formación docente), así como la actividad y dedicación investigadora (y su contribución al desarrollo, científico, tecnológico o artístico del personal docente), serán criterio relevante, atendida su oportuna evaluación, para determinar la eficiencia del profesorado en el desarrollo de su actividad profesional.

- Acceso a los cuerpos de profesorado universitario

- Dos vías de acceso: funcionarial y contratada

- Dos referentes competenciales en su ordenamiento legal:

Ministerio/Consejo de Coordinación Universita-ria /ANECA

Gobiernos CC. AA./ Agencias CC.AA.

- Evaluación parcializada y pluralidad de valoración en cada una de las dos vías:

. Dos actos y momentos diferentes en cada vía de acceso

(Habilitación-Acceso y Acreditación-Acceso) y tres pro-

cedimientos diferentes (Habilitación, Acreditación y

Acceso)

. Dos Comisiones diferentes (Externa para la Habi-litación y Acreditación e Interna para el Acceso a la Universidad). Si

bien ésta última queda a criterio de la propia universidad).

Los puntos que siguen hacen referencia a los momentos de Habilitación o de Acreditación dado que el de Acceso está sin regular por las propias universidades.

- La trayectoria académica, docente e investigadora y la defensa de un Proyecto Docente como criterio primario sólo en la vía de Habilitación y para TEU, TU y CEU.

- Se elimina el requisito de defensa de un Proyecto Docente en el caso de los CU.

- Énfasis en el dominio de la materia en el caso de TEU,TU y CEU

- Exposición y debate de un tema del programa elegido por el candidato de entre tres sacados a sorteo.

. Enfasis en la investigación tanto en el caso de TU /CEU como de CU

Exposición y debate con la Comisión de un trabajo original de investigación.

- En el caso de la Acreditación, sólo se somete a evaluación no presencial la trayectoria académica, docente e investi- 
gadora si bien se pueden establecer criterios y estándares diferenciales según la tipología de contrato permanente.

- Varios hechos a considerar:

El proceso de Habilitación, por el contenido de las pruebas y naturaleza del mismo, pretende una valoración de la competencia global de los candidatos, si bien descontextualizada de la universidad de acceso. En buena lógica, las prueba(s) de Acceso deberían perseguir la valoración del candidato al perfil y tareas definidas por la universidad. El proceso de Acreditación, por la naturaleza de las evidencias que evalúa, dista mucho de poder asegurar la competencia global del candidato. En consecuencia, la naturaleza de la(s) prueba(s) de Acceso debiera ser diferente de las de Acceso en la vía funcionarial.

Sólo en el caso de optar por una diferente valoración y consideración de ambas categorías de profesorado (minusvalorando el contratado), podría rechazarse la consecución de una equivalencia global entre los procesos Habilitación - Acceso y Acreditación - Acceso.

Se precisa la concreción por parte de las Agencias de las CCAA del contenido, criterios y estándares de los procesos evaluativos de acreditación anteriormente reseñados.

- Retribuciones al profesorado: incentivos por docencia, investigación y gestión

- Referentes competenciales en su determinación:

- Con relación a la tipología y características del complemento, y tanto para el profesorado contratado como funcionario, tienen competencias el Gobierno y las CC. AA. (art.. 54.2 y 69.2)

- Con relación a la evaluación de los méritos al respecto, y tanto para el profesorado contratado como funcionario, corresponde a la ANECA o a las CC.AA. (art. 55.4 y 69.4)

- A la vista de algunas normativas aparecidas en las CC. AA se puede observar:

Diferencias en las tipologías y características de complementos (tipo, número, periodo y cantidad)

Evaluación externa sobre C.V.

Diferenciación o no de procedimiento para investigación, docencia y gestión.

\section{Los marcos evaluativos profesionales}

El reciente y nuevo marco de referencia para la evaluación de los programas de formación médica (WFME, 2003) no deja lugar a duda. Por lo que respecta a la formación inicial, se proponen unos estándares de evaluación que se articulan en 9 áreas y 36 subáreas. El área 5, con sus dos subáreas, corresponde a la de profesorado (Academic Staff/Faculty). He aquí su contenido (o.c.:14)

\subsection{Recruitment Policy}

Basic standard: The medical school must have a staff recruitment policy which outlines the type, responsibilities and balance of academic staff (medical and non-medical / full-time and part-time) required to deliver the curriculum adequately.

Quality development: A policy should be developed for staff selection criteria, including scientific, educational and clinical merit, relationship to the mission of the institution and issues of local significance.

(Annotation: *Merit can be measured by formal qualifications, professional experience, research output, teaching experience, peer recognition, etc.)

\subsection{Staff Policy and Development}

Basic standard: The medical school must have a staff policy, which addresses a balance of capacity for teaching, research and service functions, and ensures recognition of meritorious academic activities (by reward, promotions and/or remuneration), with appropriate emphasis on both research attainment and teaching qualifications.

Quality development: The staff policy should include teachers training and development and teacher appraisal. Teacher-student ratios relevant to various curricular components and teacher representation on relevant bodies should be taken into account.
La conclusión parece obvia: una institución ( universidad facultad) ha de disponer y aplicar una política de evaluación comprensiva de su profesorado y en la que la de tomarse en consideración tanto la propia misión de la institución como las particularidades del contexto de aplicación.

\section{B. La concreción de una propuestas}

En esta segunda parte proponemos un intento de armonizar la situación jurídica descrita con un enfoque evaluativo que responda a los verdaderos retos que la evaluación del profesorado ha de afrontar: todo planteamiento de evaluación personal ha de contribuir a la mejora de la propia acción docente.

Recogemos algunos de los puntos más significativos asumidos en el Seminario sobre Evaluación del Profesorado (Cádiz, septiembre del 2002) que, organizado por las Agencias de la Calidad de Andalucía y Cataluña, pretendía constituirse en referente de las acciones evaluativas a desarrollar por los diferentes agentes institucionales.

1. ese a que el art. 55.4 de la LOU hace referencia exclusiva a la previa valoración de los méritos para la asignación de complementos retributivos, parece adecuado considerar que esta situación de evaluación debe integrarse en un marco más amplio que permita configurar un verdadero modelo de evaluación orientado también a la generación de propuestas de oportunidades de promoción y, especialmente, de mejora de las propias funciones académicas.

2. Un modelo comprensivo de evaluación requiere estar contextualizado en la realidad de cada universidad y sistema universitario y exige definir el contenido del mandato institucional a desempeñar por el profesorado y los criterios de calidad que se aplicarán. El cumplimiento de los requisitos establecidos deberían asegurar al profesorado el logro de los incentivos y/o oportunidad de promoción, sin considerar criterio restrictivo alguno. El mandato institucional debería considerar la importancia de las funciones de investigación y docencia así como la de gestión e implicación en las metas y objetivos de la universidad, centro o departamento.

3. Por lo que respecta a la función docente, parece difícil poder hacer viable un modelo de evaluación orientado a la mejora sin la correspondiente corresponsabilidad de universidades y administración en la generación de oportunidades de formación continua y de un programa de apoyo a la innovación docente. Esta debería ser considerada como una de las estrategias más eficaces en la promoción de la excelencia.

4. Establecido el marco general de evaluación por la administración competente, parece adecuado, razonable y efectivo que el modelo operativo de evaluación sea asumido y ejecutado por la propia universidad, siendo sometido, al igual que cualquier otro programa o servicio, a un proceso de acreditación. Especial atención se prestará al criterio de factibilidad del modelo propuesto, considerando su carácter progresivo a lo largo de un determinado periodo de tiempo.

5 . Acorde con los enfoques actuales de evaluación del profesorado, parece adecuado considerar al profesor como el agente principal del proceso de evaluación, tanto por lo que se refiere a la aportación de evidencias (con la consiguiente exigencia de que le sean aportadas desde diferentes fuentes), como por la necesaria valoración de su propia actividad a la luz de los objetivos propuestos y asumidos por la institución. Le evaluación de portafolio o dossier académico puede considerarse como una buena estrategia evaluativa, tanto desde la perspectiva de decisión como de orientación de la mejora.

6. La naturaleza de la función académica, la cultura profesional que traspasa los marcos geográficos y los propios planteamientos teóricos y técnicos del ámbito disciplinar de la evaluación, ponen de manifiesto la absoluta necesidad de considerar la evaluación por colegas (peer review) como la vía más idónea de implementar un modelo de evaluación. Parece razonable asumir, al igual que en otros muchos campos profesionales, que la integración de las múltiples evidencias y fuentes en orden a la emisión de un juicio de valor, debe ser realizada por profesionales expertos de la actividad académica universitaria. 
Cuadro 2. Dimensiones e indicadores de evaluación (Acreditación - Acceso)

\section{Fase externa de Acreditación}

\section{Experiencia investigadora}

1.1. Participación en proyectes de investigación obtenidos en convocatorias públicas competitivas, contratos con empresas o administraciones públicas. Realizados con otras ayudas

1.2. Publicaciones, artículos, libros, Informes de investigación y otras publicaciones

1.3. Patentes, modelos de utilidad y otros resultados

1.4. Premios y reconocimientos científicos

1.5. Estancias en el extranjero con ayudas obtenidas en programas competitivos, ayudas institucionales. Otras situaciones

1.6. Contribuciones en congresos de carácter internacional, nacional y regional

1.7. Otros méritos relevantes de investigación: tesis, trabajos de investigación en el $3 r$ ciclo, etc.

\section{Experiencia docente}

2.1. Trayectoria docente universitaria. Ámbito internacional, nacional, regional

2.2. Valoración de la trayectoria docente universitaria por parte de los usuarios (estudiantes y graduados) y responsables académicos (departamento, centro, otros)

2.3. Realización de proyectos de innovación docente obtenidos en convocatorias públicas competitivas, ayudas instituciones, otros

2.4. Difusión de los resultados de la innovación, artículos, libros, informes. Materiales derivados de los proyectos. Presentaciones en congresos, seminarios, talleres

2.5. Formación docente universitaria. Estancias, cursos, seminarios, talleres de formación didáctica y pedagógica

2.6. Otros méritos y materiales docentes

2.7. La visión del proceso enseñanza - aprendizaje

\section{Formación académica}

3.1. Predoctoral y Doctoral (institución, titulación, calificación) y Postdoctoral (institución, programa, periodo, temática, profesorado/colegas de referencia)

3.2. Informes valorativos (responsables, directores académicos)

\section{Experiencia y méritos profesionales (ASISTENCIA)}

4.1. Trayectoria profesional (institución, cargo, función, periodo)

4.2. Informes valorativos (responsables, dirección... de la calidad del desarrollo profesional)

\section{Servicios a la comunidad universitaria}

5.1. Gestión universitaria. Puestos unipersonales y en órganos colegiados

5.2. Comités científicos y p'rofesionales

\section{Servicios a la sociedad}

6.1. De carácter institucional

6.2. De carácter voluntario

\section{Fase interna de Acceso}

1.- Dominio del campo disciplinar del Área de referencia (perfil disciplinar)

2.- Dominio del diseño y de proyectos docentes

3.- Dominio de la aplicación de las TIC en la docencia

4.- Dominio de estrategias de comunicación e interacción docente

5.- Dominio del diseño y desarrollo de proyectos de investigación

6.- Conocimiento específico de la universidad

\section{Dimensión institucional y de organización académica}

Planes y programas de formación del Área de referencia
7. Parece lógico y pertinente que las dimensiones de la actividad académica a evaluar sean:

- La actividad investigadora. Pese a la existencia de posiciones críticas, parece existir un razonable consenso en cuanto a la bondad de la práctica existente. Se sugiere se avance en una mejor contextualización en los diferentes ámbitos disciplinares y a una intensificación de la información sobre el modus operandi de las respectivas Comisiones.

- La actividad docente. Han de superarse el reduccionismo presente que sólo considera la actuación del profesor en el aula y la opinión del alumnado como los elementos a considerar en la evaluación docente del profesorado.

- La actividad de gestión. Parece lógico distinguir entre las actividades de gestión unidas a cargos unipersonales reconocidos en el marco institucional de la universidad (electivos o no)y aquellas otras actividades de gestión inherentes a la propia actividad docente (deberes/tareas del profesorado).

Desde la fecha de celebración del Seminario (septiembre 2002) hemos visto aparecer los desarrollos normativos de Habilitación, Acreditación del profesorado y de Incentivos retributivos (ANECA y Agencias Autonómicas), pero no disponemos de información (en el momento de redactar esta ponencia) de ningún planteamiento global de evaluación de una universidad. Lo que sigue a continuación responde en parte (la extensión de este trabajo impide un mayor detalle) a los trabajos y propuestas realizadas desde la AQU Catalunya (1) con el objetivo de:

a) Armonizar las fases de Acreditación y Acceso a la Universidad por la vía contractual permanente (potenciada por la propia Llei d'Universitats Catalanes). El cuadro 2 explicita las dimensiones e indicadores a tener en cuenta en ambos procesos (se concreta en la figura del contrato de doctor permanente).

b) Constituirse en marco de referencia para la elaboración de un Manual de evaluación del profesorado universitario por cada una de las universidades públicas catalanas. A diferencia de otras Agencias Autonómicas, la AQU Catalunya ha asumido el punto 4 del Seminario de Cádiz y, a partir de una Guía, cada universidad propondrá un modelo de evaluación que toma en consideración el contenido del cuadro 3. Este contenido será plenamente operativo en el 2006. Los modelos propuestos serán debidamente acreditados para la fase experimental, primero, y por periodos quinquenales a partir del 2006.

Finalmente, en el cuadro 4 hacemos una propuesta académica a tomar en consideración por las Comisiones que en una fase $\mathrm{u}$ otra han de valorar la propuesta de plan docente que ha de contener el Proyecto Docente que se presenta. Es de todos sabido que los momentos de rendición de cuentas (acceso o incentivos) presentes en el actual ordenamiento legal son especialmente propicios y motivadores para afrontar un trabajo de reflexión y de adquisición de nuevas competencias por parte de los candidatos. Por lo que respecta al diseño de planes docentes, sería negativo que una vez más se perdiera la situación de presión que supone preparar un Proyecto Docente, y no se generara una cultura del hacer bien un plan docente (no olvidemos la inminente situación de convergencia europea en el diseño de programs y planes de formación).

Sin duda alguna, la aplicación de los principios de transparencia y trazabilidad de los procesos evaluativos exigirá a las Comisiones de Habilitación y Acceso una más clara explicitación de los criterios y estándares de calidad de un Proyecto Docente, así como su ponderación de valoración en el amplio contenido de dimensiones e indicadores considerados. En esta nueva etapa que se inicia, los procesos de metaevaluación deberán hacerse presentes ya que una mayor conciencia de los derechos y deberes de evaluados y evaluadores es totalmente necesaria para hacer de la evaluación un factor de calidad personal e institucional y no una partida de poker en la que los segundos juegan con cartas ocultas o marcadas. 
Cuadro 3. Dimensiones e indicadores de evaluación (Incentivo docente) Propuesta AQU Catalunya. Total implementación en el 2006

\section{Valoración del autoinforme}

1.1. Valoración docente del quinquenio

1.2. Valoración de la actividad docente del último año

1.3. Valoración de los resultados académicos

1.4. Valoración de la satisfacción de los estudiantes

1.5. Aportaciones de especial significación

1.6. Valoración de la situación del contexto y prospectiva de mejora

2. Valoración del encargo de actividad docente (del quinquenio) 2.1. Valoración de la docencia

2.2. Valoración de les tareas relacionadas con la docencia

2.3. Valoración del cumplimiento del encargo docente

\section{Valoración de la planificación docente}

3.1. Valoración del diseño del programa

3.2. Valoración de la adecuación del programa

3.3. Valoración de los recursos y material docente

\section{Valoración del desarrollo y actuación profesional}

4.1. Valoración de la formación (recibida e impartida)

4.2. Valoración de la observación directa de la actuación docente

4.3. Valoración de proyectos específicos $d$ e innovación

4.4. Valoración de la participación en tareas de mejora de la docencia
4.5. Valoración de las tareas de transición al espacio europeo

5. Valoración de los resultados de la actividad docente 5.1. Valoración de los resultados académicos

6. Valoración de la satisfacción de los estudiantes y graduados

6.1. Encuestas institucionales de valoración docente de los estudiantes

6.2. Informes o actas de grupos ad hoc

6.3. Valoraciones diferidas de estudiantes y graduados

\section{Valoración de promoción, evaluación y difusión de} la calidad docente

7.1. Valoración de la participación como docente

7.2. Valoración de la participación como experto (interno/externo) de evaluación

7.3. Valoración de la participación en la transición secundaria-universidad

8. Valoración del reconocimiento externo de la calidad docente 8.1. Valoración de las publicaciones docentes

8.2. Valoración de los premios y distinciones docentes

8.3. Valoración de la invitación docente en otras universidades

Cuadro 4. Check-list para la evaluación del plan docente de un Proyecto Docente

\section{A. Programa docente}

- Existe claridad y coherencia en el propósito y los objetivos del curso con relación al Programa o Plan de Estudios de la universidad

- Establece una secuencia lógica en la organización de los contenidos y concreción en los objetivos de Aprendizaje

- Presenta diversas experiencias de aprendizaje para el logro efectivo de los objetivos

- Se integran las TIC como soporte a la enseñanza

- Considera el uso de materiales didácticos diversos

- Propone una evaluación amplia y permanente de los logros del aprendizaje mediante múltiples estrategias

- Establece una demanda de trabajo (para el estudiante) en congruencia con las características de la materia

\section{B. La investigación en el proceso de enseñanza - aprendizaje}

- En el programa del curso se especifica la realización de actividades o proyectos de investigación con los estudiantes

- Aborda enfoques/planteamientos para orientar a los estudiantes en la elaboración de proyectos de investigación.

- En el contenido del Proyecto Docente se refleja la trayectoria investigadora del profesor

- Presenta alguna actividad innovadora dentro del proyecto docente a desarrollar.

- Hace referencia a fuentes documentales sobre la enseñanza en el campo disciplinar que le compete

\section{Actividad tutorial}

- Considera un plan sistemático de acción tutorial y de supervisión del aprendizaje del estudiante

Bibliografía

Arreola, R.A. (2000) Developing a Comprehensive Faculty Evaluation System $\left(2^{\text {nd }}\right.$ Ed).

Bolton, MA: Anker Pub. Co.

Fielden, J. (2001) Higher Education Staff Development: Continuing Mission. Thematic Debate of the Follow-up to the World Conference on Higher Education. UNESCO (www.unesco.org)

Millis, B.J. (1994) Faculty Development in the 1990s: What It Is and Why We Can't Wait. Journal of Counseling and Development, 72, 454-464
Rodríguez, S. (2003) Nuevos retos y enfoques en la formación del profesorado universitario. Revista de Educación (En prensa)

Schuster, J.H. (1993) The Environment for Faculty and Their Development as Teachers.

In: M. Weimer (ed.) Faculty as Teachers. University Park, PA: National Center on Postsecondary Teaching, Learning, and Assessment. (pp.109113)

Word Federation for Medical Education (WFME, 2003) Basic Medical Education. WDMD Global Standards for Quality Improvement. WFME Office: University of Copenhagen. 